\title{
VALIDEZ DE CONSTRUCTO Y CONFIABILIDAD DE LA ESCALA DE AUTOCONCEPTO SOBRE LAS HABILIDADES DE M. C. DAYTON
}

\author{
César Merino Soto ${ }^{1}$ \\ Maritza Díaz Casapía ${ }^{2}$
}

El presente artículo reporta sobre la validez de constructo y confiabilidad interna de la escala de autoconcepto sobre las Habilidades (Dayton, 1968), que mide la percepción de las propias habilidades académicas en los cursos escolares, en base a las comparaciones que hacen los contra referentes externos. Recientemente, el modelo propuesto por Marsh y Craven (2002), sobre el autoconcepto académico construido basado en el marco de referencia externo dirige el objetivo del presente estudio, debido a su relación directa entre este modelo y el instrumento de Dayton. Aplicando un enfoque factorial confirmatorio con factores correlacionados, en una muestra de 97 alumnos, los hallazgos preliminares señalan que el modelo multidimensional se ajusta adecuadamente. La confiabilidad de la subescalas es bastante óptima. Aunque estos resultados sugieren estabilidad en la solución factorial, se requieren estudios de intervalidación de este potencial instrumento.

PALABRAS CLAVE: Autoconcepto académico, análisis factorial, habilidades

The present article report about the construct validity and internal reliability of the Self concept of Ability scale (Dayton, 1968), that measure the perception of the student's academic abilities in the school class, it based on the comparisons that make those against references external. Recently, the theoretical model proposed by Marsh and Craven (2002), on the selfconcept academic based on the external references, it directs the objective of the present study, because to their direct relationship between this model and the instrument of Dayton. Applying a confirmatory factorial approach with correlated factors, in a sample of 97 students, the preliminary findings point out that the multidimensional model is fit appropriately to the data. The reliability of the subescales is quite good. Although these results suggest stability in the factorial solution, studies of corss-validation of this potential instrument are required.

KEYWORDS: academic selfconcept, factorial analysis, abilities

${ }^{1,2}$ Servicio de Psicología, Defensoría Municipal del Niños y del Adolescente, Av. José Olaya 166 (Casa de la Cultura), Lima 9 - Chorrillos. 


\section{INTRODUCCIÓN}

El autoconcepto puede ser visto como un atributo multifacético, cuyas facetas provienen de la percepción el sujeto en áreas de desarrollo relevantes. Además, es uno de los constructos más importantes de las ciencias sociales (Marsh \& Craven, 2002). Ha tendido a ser utilizado intercambiablemente con otro constructo relacionado, la autoestima (García, 1995), que es la valoración emocional del autoconcepto y están vinculados estrechamente a los sentimientos y el desarrollo de la personalidad.

Las investigaciones del autoconcepto en escolares indican consistentemente que el nivel de autoconcepto esta en directa proporción a su rendimiento académico y al desarrollo de habilidades sociales, como asertividad, mejor comunicación con pares, consecución de metas a corto plazo. También, se lo ha identificado dentro de una variedad de factores específicos que pueden contribuir al estrés del estudiante (Matheny, Aycock, Pugh, Curlette, \& Silva, 1986). Por ejemplo, el bajo autoconcepto parece ser tanto la causa y consecuencia de un pobre desempeño académico (Davidson \& Lang, 1960)

Se ha demostrado que el desarrollo de la autoaceptación y autoestima depende del impacto de la calidad y naturaleza de las interacciones que ocurren entre padres e hijos, especialmente en los primeros 5 años (Banham, Hanson, Higgins, Jarret, 2000), donde se establecen los cimientos de la futura personalidad. Sin embargo, parece que el autoconcepto medido multidimensionalmente refleja más diferencias que el autoconcepto medido globalmente (Worrell, 2000; Worrell y Latto , 1999)

En la educación escolar tanto el autoconcepto como la autoestima se encuentra presente en todas las experiencias de enseñanza-aprendizaje, llegando inclusive a señalar que es su principal fuente de desarrollo específicamente se expresa en el rendimiento académico, en la motivación, las actitudes y las relaciones interpersonales entre el profesor y los alumnos en general.

Unos de los aspectos cuya investigación parece estar emergiendo, es la relación entre autoconcepto académico y variables externas con textuales. Uno de los componentes del autoconcepto parece ser las comparaciones entre el propio desempeño y el de otros; en este sentido, mientras más grande sea la discrepancia, las respuestas cognitivas, emocionales conductuales estarán estrechamente vinculadas. La evaluación cognitiva del desempeño propio comparándolo con los demás es un aspecto también de una orientación competitiva en el rendimiento, que ha sido vinculado a los futuros logros. Considerando la formulación teórica actual de Marsh y Craven (2002) en la formación del autoconcepto académico, un papel prominente proviene de procesos de comparación social que hace el sujeto de las propias habilidades y de las de los demás. El marco de referencia externo tiene un impacto considerable en el rango que alcanza el autoconcepto académico (Marsh y Craven, 2002).

Una de las barreras que podría impedir la investigación dentro del marco de Marsh y Craven (2000) es la carencia de una medida que recoja estos procesos. Mitchell Dayton profesor de la Universidad de Maryland, USA ha desarrolló una escala de autoconcepto de habilidades (Dayton, 1968), instrumento que ha sido utilizado en nuestra aplicación. El contenido de la escala fue generado de manera altamente sistemática. Seis contenidos de áreas académicas fueron identificadas como importantes en los niveles elementales y secundarios de la escolaridad: Aritmética, Lenguaje, Estudios Sociales, Ciencias, Música y Arte. Estas subescalas forman seis áreas en que la percepción del estudiante sobre sus habilidades se hace estimulando procesos de comparación social con marcos de referencia externos (Marsh \& Craven, 2002). Los Ítem se presentan con referencias temporales (presente y el futuro) y contextuales específicos de las clases de la secundaria (la clase, el 
grado, grupo de amigos; las futuras clases de la secundaria, asociaciones futura), otros estudios en general y las propias habilidades. Se combinan los seis contenidos académicos con las siete bases de comparación, consiguiéndose 42 ítems utilizados en la construcción de la prueba.

El objetivo del presente estudio es obtener información preliminar sobre las características psicométricas del instrumento, mediante un acercamiento analítico a su dimensionalidad y consistencia interna.

\section{MÉTODO}

\section{Muestra}

La muestra proviene de los hijos de los empleados de una institución de seguro médico a nivel nacional, inscritos en un programa de recreación vacacional durante los meses de Enero y febrero. Los niños y adolescentes asistían tres veces por semana, y sus actividades incluían deportes a nivel recreativo y talleres de habilidades sociales. De acuerdo a los informes del servicio social, todos los padres varones y mujeres de la institución tienen educación superior completa, y envían a sus hijos mayoritariamente a colegios de gestión particular.

Se procesaron 97 casos para el análisis.

La edad media de los alumnos evaluados fue 12.3, con una desviación estándar de 1.11. La edad mínima de 10 años y una máxima de 15 años. El rango de grados de los alumnos fue desde 5to de primaria hasta 4to de secundaria. 12 niños no reportaron su grado. La distribución del género fue balanceado, teniendo 47 varones (48.5\%) y 50 mujeres (51.5).

\section{Procedimiento}

La aplicación del instrumento se llevó a cabo en un club recreacional, en la Ciudad de Lima. Durante la ejecución de los talleres de habilidades sociales, los participantes recibieron el cuestionario para ser llenado sin límites de tiempo. La evaluación se realizó en grupos de 20 participantes, de distribución mixta entre hombres y mujeres. La aplicación fue estándar para todos los grupos y los asistentes de evaluación se aseguraron de atender las dudas suscitadas sobre el instrumento. En general, hubo pocas dudas sobre el llenado de las respuestas por parte de los participantes.

\section{Instrumentos}

Prueba de Autoconcepto Académico.

Dayton (1968) elaboró la prueba como uno de los componentes del estudio para mejorar los servicios educativos de su distrito escolar; unos de los criterios fue explorar la percepción de los niños como estudiantes. Contiene 49 ítems y las opciones de respuesta va desde 1 (opción negativa) hasta 5 (opción positiva). En una muestra grande (2204 estudiantes desde el 3ro al6to grado), el análisis por componentes principales y rotación varimax reveló consistentemente 7 factores que agrupaban Ítems según lo hipotetizado (Lenguaje, Matemáticas, Ciencias, Música, Educación Física, Historia, Arte), excepto los Ítems que pretendían medir una apreciación general del autoconcepto. Esta estructura fue intervalidada en las diferentes submuestras de grado escolar que componían la muestra en el estudio original. La confiabilidad interna, evaluada por el método de mitades, arrojó coeficientes de bajos para el puntaje General (.37 hasta .72), pero generalmente altos para el resto de las escalas (mayormente entre .80 y.90).

\section{ANÁLISIS}


Debido a que la razón entre la cantidad de sujetos y las variables analizadas (ítem de la escala) no satisface los criterios usualmente declarados para un análisis factorial (MacCallum, Widaman, Zhang, \& Hong, 1999), los datos se trabajaron preliminarmente con el análisis de clusters, para explorar la estabilidad de la estructura de acuerdo a lo definido por Dayton (1968). El análisis de clusters es un método recomendado para hacer frente a situaciones como la nuestra (Aldenderfer \& Blashfield, 1984; Moreno \& PérezGil, 1993) y actualmente se ha reportado su uso exitoso para confirmar la bidimensionalidad de un auto-reporte sobre la crianza (ver Merino, Cohen \& DÍaz, 2003; Merino \& Cohen, 2003). La línea confirmatoria del estudio buscó comprobar la estructura factorial del instrumento, previamente establecida por Dayton (1968); así que se utilizó una técnica del análisis factorial confirmatorio mediante el programa CONFA (McDonald, 1999), permitiendo que los factores sean correlacionados. La matriz de patrón factorial, que define la ubicación de los Ítems en sus supuestas subescalas fue la matriz sobre la que se efectuó la rotación. Los resultados del análisis de cluster y del análisis factorial coincidieron perfectamente, agrupando los Ítems de manera idéntica; además, las cargas factoriales $(>0.35)$ y comunalidades (generalmente $>0.5)$ generadas del análisis factorial fueron consideradas lo suficientemente altas y óptimas cuando se tienen tamaños muestrales de cercanos a 100 (Guadalogni \& Velicer, 1988; MacCallum, Widaman, Zhang, $\&$ Hong, 1999). Esto sugiere que se pueden extraer conclusiones moderadamente estables con los datos, desde una vertiente analítica factorial. Únicamente los resultados analíticos factoriales se presentarán.

El examen de las diferencias entre la muestra masculina y femenina sobre cada una de las subescalas se hará con la prueba $t$ para muestras independientes y se estimará la magnitud del efecto de estas diferencias con la $d$ de Cohen (Coe \& Merino, 2003). La consistencia interna se verificará mediante el coeficiente de confiabilidad (Cronbach, 1951), Y la correlación ítem-test corregida (Navas, 2001).

\section{RESULTADOS}

La Tabla 1 muestra los resultados del análisis factorial, con rotación basada en la matriz de hipotetizada. Los 7 factores contaron con el 59\% de la varianza. Los Ítems se ajustaron perfectamente a sus respectivas subescalas, con el primer factor conteniendo los Ítems de matemáticas; así que este factor puede ser nombrado Matemáticas, con cargas factoriales entre .88 y .76. Los siguientes factores extraídos y etiquetados fueron Ciencias (.89 a .76), seguido de Música (.75 a .89), Lenguaje (.67 a .86), Educación Física (.65 a .91), Historia (.70 a .88) y General (.36 a .69). Este último factor se ajustó pobremente a sus Ítems en comparación a las restantes subescalas. Claramente, este factor no está adecuadamente definido por los Ítems con lo que se esperaba estar compuesto.

De la matriz de discrepancias, el residual más grande fue .22 , y el residual promedio 05 . La raíz media cuadrática (RMS) fue de .07, que es un valor marginalmente aceptable. El índice de bondad de ajuste de Tanaka alcanzó un valor de .96. Estos indicadores de ajuste nos proveen los fundamentos cuantitativos para considerar el modelo propuesto de 7 factores como consistente. Debido que el factor «General» no se ajustó como se esperaba, se analizó nuevamente con un modelo establecido en 6 factores, excluyendo el factor «General». Sólo una ligera mejora se apreció, con RMS de .06 y un Índice de Tanaka de .97. Así que, el modelo de 7 factores parece ajustarse apropiadamente a los datos y retener suficiente varianza de los constructos explorados. 
Tabla 1: Análisis factorial confirmatorio (con rotación oblicua): Cargas de patrón

\begin{tabular}{|c|c|c|c|c|c|c|c|c|}
\hline & Matem. & Cencias & Música & Lenguaje & Ed Física & Historia & General & $h^{2}$ \\
\hline $\begin{array}{l}\mathrm{m} 38 \\
\mathrm{~m} 14\end{array}$ & $\begin{array}{l}0.76 \\
0.78\end{array}$ & & & & & & & 059 \\
\hline m8 & 0.79 & & & & & & & $\begin{array}{l}0.61 \\
0.63\end{array}$ \\
\hline $\mathrm{m} 20$ & 0.82 & & & & & & & 0.68 \\
\hline $\mathrm{m} 32$ & & & & & & & & 0.69 \\
\hline $\mathrm{m} \times 44$ & 0.84 & & & & & & & 0.71 \\
\hline $\mathrm{m} 26$ & & & & & & & & 0.77 \\
\hline 047 & & 0.76 & & & & & & 0.58 \\
\hline 041 & & 0.81 & & & & & & 0.65 \\
\hline c35 & & 0.86 & & & & & & 0.74 \\
\hline c11 & & 0.88 & & & & & & 0.78 \\
\hline c23 & & & & & & & & 0.77 \\
\hline $\mathrm{c} 17$ & & 0.89 & & & & & & 0.80 \\
\hline$c 29$ & & & 0.75 & & & & & 0.80 \\
\hline 042 & & & & & & & & 0.57 \\
\hline u36 & & & $\begin{array}{l}0.78 \\
0.79\end{array}$ & & & & & 0.60 \\
\hline u12 & & & $\begin{array}{l}0.79 \\
0.83\end{array}$ & & & & & 0.62 \\
\hline u48 & & & 0.83 & & & & & 0.69 \\
\hline ul8 & & & 0.84 & & & & & 0.71 \\
\hline 1024 & & & 0.84 & & & & & 0.70 \\
\hline u30 & & & 0.89 & & & & & 0.79 \\
\hline 139 & & & & 0.67 & & & & 0.46 \\
\hline 145 & & & & 0.74 & & & & 0.55 \\
\hline 19 & & & & 0.8 & & & & 0.64 \\
\hline 133 & & & & 0.81 & & & & 0.66 \\
\hline 115 & & & & 0.82 & & & & 0.68 \\
\hline 121 & & & & 0.82 & & & & 0.67 \\
\hline L27 & & & & 0.86 & & & & 0.75 \\
\hline$e 43$ & & & & & 0.65 & & & 0.43 \\
\hline e37 & & & & & 0.73 & & & 0.54 \\
\hline 013 & & & & & 0.74 & & & 0.56 \\
\hline$e 25$ & & & & & 0.82 & & & 0.67 \\
\hline e19 & & & & & 0.84 & & & 0.71 \\
\hline e49 & & & & & 0.85 & & & 0.72 \\
\hline e31 & & & & & 0.91 & & & 0.84 \\
\hline $\mathrm{H} 40$ & & & & & & 0.7 & & 0.49 \\
\hline h10 & & & & & & 0.73 & & 0.54 \\
\hline H46 & & & & & & 0.73 & & 0.53 \\
\hline H34 & & & & & & 0.74 & & 0.55 \\
\hline hl6 & & & & & & 0.77 & & 0.59 \\
\hline $\mathrm{h} 22$ & & & & & & 0.88 & & 0.77 \\
\hline h28 & & & & & & 0.88 & & 0.78 \\
\hline 84 & & & & & & & 0.36 & 0.13 \\
\hline $8^{3}$ & & & & & & & 0.37 & 0.14 \\
\hline g? & & & & & & & 0.4 & 0.16 \\
\hline g1 & & & & & & & 0.45 & 0.20 \\
\hline gs & & & & & & & 0.45 & 0.20 \\
\hline$\frac{82}{86}$ & & & & & & & $\begin{array}{l}0.52 \\
0.69\end{array}$ & $\begin{array}{l}0.27 \\
0.48\end{array}$ \\
\hline Min & .76 & .76 & .75 & 67 & 65 & .70 & 36 & \\
\hline Máx. & .88 & .88 & 88 & 86 & 91 & 88 & 69 & \\
\hline
\end{tabular}


La confiabilidad por consistencia interna reveló coeficientes alfa altos, sugiriendo un estrecho rango de error de medición; así que, esta evidencia preliminar indica que las subescalas proveen resultados bastantes confiables y con posibles usos diagnósticos (Barker, Pistrang, \& Elliot, 1994). Estas estimaciones se hallan en la Tabla 2. Respecto al rango de correlaciones Ítem-test, estas fueron elevadas como era de esperar; la excepción fue con la subescala General, que obtuvo los coeficientes más bajos.

\section{Tabla 2: Consistencia interna (alfa y correlación Ítem-test corregida) y Media, Subescalas Subescala y d de Cohen, en las Subescalas}

\begin{tabular}{|c|c|c|c|c|c|c|c|c|c|}
\hline & \multirow{2}{*}{$\alpha$} & \multicolumn{3}{|c|}{ Rite $^{1}$} & \multirow{2}{*}{$\frac{\text { Varones }}{\text { Meda (de) }}$} & \multirow{2}{*}{$\begin{array}{c}\text { Mujeres } \\
\text { Media(de) }\end{array}$} & \multirow{2}{*}{$\frac{\text { Total }}{\text { Media(d.e.) }}$} & \multirow{2}{*}{$\stackrel{f}{(g 1)}$} & \multirow{2}{*}{$d$ Cohen } \\
\hline & & Min & Media & Max. & & & & & \\
\hline Matemáticas & 93 & 0.76 & 0.78 & 0.86 & $\begin{array}{l}25.6 \\
(6.5)\end{array}$ & $\begin{array}{l}27.0 \\
(6.9)\end{array}$ & $\begin{array}{l}26.3 \\
(6.7)\end{array}$ & $\begin{array}{l}.964 \\
909\end{array}$ & -0.20 \\
\hline Leniguajo & 92 & 0.65 & 0.77 & 0.80 & $\begin{array}{l}27.0 \\
(5,1)\end{array}$ & $\begin{array}{l}28.5 \\
(5.4)\end{array}$ & $\begin{array}{l}27.8 \\
(5.2)\end{array}$ & $\begin{array}{l}-1.31 \\
(88)\end{array}$ & -0.28 \\
\hline Historia & 92 & 0.72 & 0.77 & 0.81 & $\begin{array}{l}26.2 \\
(5.1)\end{array}$ & $\begin{array}{l}28.8 \\
(49)\end{array}$ & $\begin{array}{l}27.5 \\
(5.1)\end{array}$ & $\begin{array}{c}-2.36^{*} \\
(88)\end{array}$ & .0 .50 \\
\hline Ciencias & 95 & 0.82 & 0.83 & 0.88 & $\begin{array}{l}27.8 \\
(6.4)\end{array}$ & $\begin{array}{l}28.2 \\
(6.5)\end{array}$ & $\begin{array}{l}28.0 \\
(6.4)\end{array}$ & $\begin{array}{l}.320 \\
(89)\end{array}$ & -0.07 \\
\hline Música & 95 & 0.80 & 0.82 & 0.89 & $\begin{array}{l}263 \\
(68)\end{array}$ & $\begin{array}{l}30.2 \\
(4.8)\end{array}$ & $\begin{array}{l}28.4 \\
(6.0)\end{array}$ & $\begin{array}{l}-2.94 * * \\
(61.3)\end{array}$ & -0.69 \\
\hline $\begin{array}{l}\text { Educación } \\
\text { Fisica }\end{array}$ & 93 & 0.67 & 0.79 & 0.89 & $\begin{array}{l}28.4 \\
(5.9)\end{array}$ & $\begin{array}{l}29.4 \\
(5.6)\end{array}$ & $\begin{array}{l}289 \\
(5.7)\end{array}$ & $\begin{array}{l}.809 \\
(91)\end{array}$ & -0.17 \\
\hline General & 66 & 0.24 & 0.36 & 0.49 & $\begin{array}{l}26.5 \\
\text { (3.1) }\end{array}$ & $\begin{array}{l}279 \\
(3.7)\end{array}$ & $\begin{array}{l}27.2 \\
(3.4)\end{array}$ & $\begin{array}{c}-2.02 * \\
(95)\end{array}$ & .0 .41 \\
\hline
\end{tabular}

1 Correlación item-test corregida

$* p<01 ; * p<.05$

Al probar las diferencias entre los puntajes de acuerdo al género de los estudiantes, se observa en la Tabla 2 una tendencia constante de puntajes elevados en las mujeres sobre los varones. También se observa una secuencia en las magnitud del efecto, que va desde muy bajo $(\mathrm{d}=-0.07 \mathrm{y}-\mathrm{D} .17)$ y bajo $(\mathrm{d}=0.20 \mathrm{y}-0.28)$, hasta un nivel moderado $(\mathrm{d}=0.50 \mathrm{y}-$ 0.69). De estas diferencias, sin embargo, únicamente han sido estadísticamente significativas en la subescala Historia, $\mathrm{t}(88)=-2.362, \mathrm{P}<0.05, \mathrm{~d}=-.050$; y Música,t (1.63) $=-2.946, \mathrm{p}<.01, \mathrm{~d}=\cdot 0.69$. La tabla 3 muestra la información escrita en este párrafo.

Las correlaciones entre las subescalas, en general, alcanzan niveles moderados y significativamente estadísticos. Esto sugiere una relativa oblicuidad entre los componentes, pero se observa que hay correlaciones que van desde una baja a moderada varianza compartida; por ejemplo, entre Ciencias y Lenguaje, la varianza compartida entre ellas es de 41 $\%$. Algunas de estas correlaciones sugieren, sin embargo, asociaciones oblicuas 
Tabla 3: Correlaciones r de Pearson entre las Subescalas

\begin{tabular}{|c|c|c|c|c|c|c|}
\hline & Matemáticas & Lenguaje & Historia & Ciencias & Música & Educación Fisica \\
\hline Matemáticas & 1.000 & & & & & \\
\hline Lenguaje & $505 * *$ & 1.000 & & & & \\
\hline Historia & $252^{*}$ & $544 * *$ & 1.000 & & & \\
\hline Ciencias & $521 * *$ & $642 * *$ & $415 * *$ & 1.000 & & \\
\hline Música & $252^{*}$ & 215 & $482^{* *}$ & $363^{* *}$ & 1.000 & \\
\hline Educación Física & $317^{* *}$ & $228^{*}$ & 169 & $236^{*}$ & $258 *$ & 1.000 \\
\hline
\end{tabular}

\section{DISCUSIÓN}

Este estudio sirve como un importante paso para la inclusión de una herramienta sobre el autoconcepto de las habilidades en los estudiantes. La estructura hallada confirma la elaborada originalmente por Dayton (1968) en sus estudios con estudiantes escolares norteamericanos, lo cual aporta información inter-cultural al respecto, junto al hecho que es posible explorar aspectos específicos del autoconcepto sobre las habilidades, basándose en la percepción comparativa del propio sujeto con el resto de alumnos en una muestra de latinoamericanos.

Una discusión sobre los aspectos metodológicos presentes en nuestro estudio merece algunos párrafos. De acuerdo a algunos autores (Fabrigar et al., 1999; Wegener \& Fabrigar, 2000), no podríamos hacer inferencias apropiadas sobre la estructura latente desde la aplicación del análisis factorial, debido a los supuestos estadísticos relacionados con el tamaño muestral. Sin embargo, la magnitud de las cargas y comunalidades halladas asegura que los resultados del análisis se podrán reproducir de manera consistente en otras muestras o con la aplicación de otros modelos factoriales (Velicer \& Jackson, 1990). Pero más importante aún, lo que queda en la agenda de futuras investigaciones es la necesaria validación de los presentes resultados en términos de replicación (Hair, Anderson, Tatham \& Black, 1999). La validación de nuestros resultados requerirá una mayor muestra y la búsqueda de la convergencia con otras medidas de autoconcepto multidimensional. Es de notar que las altas cargas y comunalidades dan expectativas favorables para la replicación futura de nuestros resultados, debido que tales magnitudes se hallan frecuentemente en instrumentos que conseguirán estabilidad en los resultados aún con tamaños muestrales similares a la nuestra (Guadalogni \& Velicer, 1988; MacCallum et al, 1999).

El autoconcepto sobre las habilidades académicas es un componente de los aspectos autoreferenciales del estudiante y su operacionalización mediante un instrumento resultará bastante promisorio, señalándose que está asociada con una reciente elaboración teórica propuesta por Marsh y Craven (2002); su formulación central es sobre el efecto que tiene la percepción de las propias habilidades y de las de otros, en un contexto educacional inmediato, sobre el autoconcepto académico. En la actualidad, la medición multidimensional del autoconcepto y su distinción de la autoestima, permite examinar y estudiar con mayor profundidad las relaciones entre autoconcepto y la salud mental, como ocurre con las conductas de salud. Algunos estudios contradicen, sin embargo, la relación entre autoconcepto y otros aspectos relacionados con la salud mental positiva, por ejemplo, los estilos de vida (Balaguer y Pastor, 2001). Balaguer y Pastor (2001) hallaron que esta 
relación es más compleja que la simple examen del autoconcepto global con conductas de salud, y la contribución de las diferentes dimensiones del autoconcepto puede explicar mejor las relaciones hipotetizadas entre estas variables.

Con la evidencia de validez de constructo presentada aquí, el instrumento puede ser considerado dentro del «arsenal» de medidas psicológicas, aunque dejamos en claro que el proceso de su investigación psicométrica aquí expuesto solo es parcial. Los aspectos que cubre el estudio de la validez de un instrumento no se agota dado los usos y los objetivos de su interpretación en diferentes situaciones (Navas, 2001), pero algunos usos se pueden identificar. Así entre sus probables usos, se tiene que: 1) puede permitir identificar estudiantes que podrían experimentar dificultades en su comportamiento académico debido a sus percepciones autoreferenciales bajas; y 2) para describir diagnósticamente el impacto de determinados eventos sobre las áreas del yo académico que requieren apoyo psicológico. Pero aunque los resultados de nuestro estudio son promisorios, animamos a los investigadores educativos a extender y replicar nuestros aportes.

\section{REFERENCIAS BIBLIOGRÁFICAS}

Aldenderfer, M. S., \& Blashfield, R. K. (1984). Cluster Analysis (Sage University Paper series on Quantitative Applications in the Social Sciences, Nro. 44). Beverly Hills, CA: Sage.

Balaguer, 1. \& Pastor, Y. (2001) Un estudio acerca de la relación entre el autoconcepto y los estilos de vida en la adolescencia media. Trabajo presentado en CIOPA 2001 Congreso Internacional Online De Psicología Aplicada, 11 al 18 de noviembre. En:http://psicologiaonline.com/ciopa200 1/index.htm

Banham, v., Hanson, J., Higgins, A. \& Jarrett, M. (2000) Parent-Child Communication and its Perceived Effects on the Young Child's Developing Self-Concept. Trabajo presentado en la 7th Australian Institute of Family Studies Conference: «Family Futures: Issues in Research and Policy», Sydney, Australia

Barker, c., Pistrang, N., \& Elliot, R. (1994). Research methods in clinical and counseling psychology. West Sussex: John Wiley \& Sons.

Coe, R. \& Merino, C. (2003) Magnitud del efecto: Guía inicial para usuarios. Revista de Psicología - Universidad Pontificia Católica del Perú, 23(1).

Cronbach, L. J. (1951). Coefficient alpha and the internal structure of test. Psychometrika, 16(3),297-334.

Davidson, H.H., \& Lang, G. (1960).

Children 's perceptions of their teachers'feelings toward them related to selfperceptions, school achievement and behavior. J oumal of Experimental Education, 29,107-118.

Dayton, M. C. (1968) Selfconcept ofability scale: Technical manual. Research and Demonstration Cen er of the Interprofessional Research Commission on Pupil Personnel Services, University of Maryland.

Fabrigar, L. R., MacCallum, R. c., Wegener, D. T. \& Strahan, E. J. (1999) Evaluating the use of exploratory factor analysis in psychological research. Psychological Methods, 4(3), 279-299.

García, A. (1995). Inventario EOS de Autoconcepto en el Medio Escolar. EOS: Madrid.

Guadalogni, E., \& Velicer, W. (1988). Relation of sample size to the stability of component patterns. Psychological Bulletin, 103(2), 265-275.

Hair, J. F, Jr., Anderson, R. E., Tatham, R. L., \& Black, W C. (1999). Análisis 
multivariante (5ta ed.). Madrid: Prentice Hall Iberia.

MacCallum, R. C., Widaman, K. F, Zhang, \& Hong, S. (1999). Sample size in factor analysis. Psychological B ulletin, 4( 1), 84-89.

Marsh, H. W \& Craven, R. G. (2002) The Pivotal Role of Frames of Reference in Academic Self-concept Formation: The Big Fish Little Pond Effect. SelfConcept Enhancement and Learning. Facilitation (SELF) Research Centre, University of Western Sydney.McDonald, R. P. (1999) Test theory: a unified treatment. Mahwah, NJ: Erlbaum.

Matheny, K. B., Aycock, D. W, Pugh, J. L., Curlette, W L. \& Silva, K. A. (1986): Stress coping: a qualitative and quantitative synthesis with implications for treatment. The Counseling Psychologist, 14(4), 449549

Merino, C., Cohen, B. \& Díaz, M. (2003) De los niños a los padres: El inventario de percepción de conductas parentales. Personas - Universidad de Lima. (En revisión)

Merino, C. \& Cohen, B. (Más) evidencias de la validezfactorial dellventario de Percepción de Conductas Parentales. Personas - Universidad de Lima. (En revisión)

Moreno, R., \& Pérez-Gil, J. A. (1993). On equivalence of factor analysis, linear discriminant analysis and .cluster analysis. In R. Steyer, K. F Wender \& K. F Widaman (Eds.), Psychometric Methodology. Proceedings of the 7th European Meeting ofthe Psychometric Sociaty in Trier (343-348). Stuttgart and New York: Gustav Fischer Verlag.

Navas, M. (2001). La fiabilidad como criterio métrico de la calificación global del test. In M. L. Navas (Ed.), Métodos, diseños y técnicas de investigación psicológica (pp. 213262). Madrid: UNED.

Nunnally, J.C. \& Bernstein, I. J. (1995).

Teoría psicométrica (3ra ed.). México, D. F: McGraw-Hill.

Velicer, W., \& Jackson, D. N. (1990).

Components analysis versus common factor analysis: Some issues in selecting an appropriate procedure. Multivariate Behavioral Research, 25(1), 1-28.

Wegener, D. T. \& Fabrigar, L. R. (2000) Analysis and design of no experimental data: Addressing causal and non causal hypotheses. En H. T. Reiss \& C. M. Judd (Eds.), Handbook of research methods in social and personality psychology. (pp. 412-450). Cambridge University Press.

Worrell, F (2000) The reliability and utility of self-concept instruments with at-risk populations. The Journal of At-Risk Issues, 5, 31-41.

Worrell, F C., Latto, I. K., Perlinski, M. A. (1999) The relationship of risk status to selfesteem and perceived life changes. The Journal of At-Risk Issues,5, 33-38. 